Balancing medical education with service in the workplace: a qualitative case study

\begin{tabular}{|r|l|}
\hline Journal: & Journal of Workplace Learning \\
\hline Manuscript ID & JWL-05-2021-0064.R1 \\
\hline Manuscript Type: & Research Paper \\
\hline Keywords: & $\begin{array}{l}\text { Workplace learning, Continuing professional development, Experiential } \\
\text { learning, Leadership }\end{array}$ \\
\hline \multicolumn{2}{|l}{} \\
\hline
\end{tabular}

SCHOLARONE ${ }^{\text {m }}$

Manuscripts 


\title{
Balancing medical education with service in the workplace: a qualitative case study
}

\begin{abstract}
Purpose: Finding a balance between the provision of quality individualized care and the ongoing education of junior doctors had been flagged as a concern at a large NHS teaching hospital in the north of England. In response to this, the organization introduced an intervention designed to improve educational culture by providing support to educators, leaders and clinical staff.

Method: This article features themed results from eight in-depth interviews with educators, consultants and junior doctors to describe and evaluate the process and impact.

Findings: Factors that contributed to a positive educational environment included: trainees and educators feeling valued, the presence of supportive leaders, and the provision of a safe space for learning. Perceived barriers included time constraints, differing motivation, and the generic format of formal education. Participants reflected on how the Wrap Around project helped improve the workplace educational culture and offered suggestions for further improvement including the provision of ongoing feedback to learners about their performance.

Originality: Research aimed at recognising and resolving the perceived tensions between the priorities of education and healthcare delivery has been flagged as a gap in the literature. We argue that developing and enhancing collaborative leadership and educational culture within an organization can reduce these tensions for those working on the front line. Future work should focus on addressing the perceived distinction between the two within services.
\end{abstract}

\section{Introduction}

Medical education relies on workplace-based experiential learning, therefore

learning and healthcare delivery must happen simultaneously and effectively in

clinical settings. It is crucial that health professionals address the goals of providing

quality healthcare and successful workplace-based learning for professionals

(Yardley et al. 2015).

This study explores an intervention designed to improve the organizational learning

culture as a way to address the balance of health professional education with patient 
care delivery. Mulder et al. (2019:36) describe organisational learning culture as 'the set of implicit messages about values, norms, and attitudes that learners infer from the behavior of individual role models as well as from group dynamics, processes, rituals, and structures'.

This area of inquiry was identified as essential in Dennis et al's work asking 1300 stakeholders in Scotland for their priorities for future research in medical education. The top priorities of learner participants were the need for research into 'balancing service and training conflicts' and 'creating an effective workplace learning culture' (2014:1082). Dennis et al advise that 'the time spent by health care practitioners in education and training can often be presented, arguably wrongly, as time away from service delivery' (2014:1079), and conclude that an effective organisational learning culture can be developed through achieving this balance. According to Sholl et al (2017) much of the work that mentions balancing learning with service is embedded in literature dealing with a wider focus, such as evaluations of clinical teaching, or studies investigating occupational stress. We address this identified gap in the literature by exploring perspectives of educators, consultants and junior doctors an NHS Trust in North of England.

Sholl et al's systematic review of literature (2017) explored how the delivery of effective service to patients can be facilitated in the workplace whilst providing opportuntities for learning. They reviewed key interventions that help achieve and maintain this balance, noting that the existing literature on balancing education with service seems to privilege formal training over informal learning - which they therefore claim reinforces the notion that education is seen as separate to service. 
It is therefore important that both learners and educators understand the significance of workplace-based learning and are motivated to make it happen. Workplace learning happens best when people are able to work alongside other workers and with clients, tackling challenging tasks, whilst being supported to give and receive feedback on their ideas (Eraut 2011). To ensure this happens, it is essential that workplace learning is given sufficient institutional and managerial backing with appropriate funding and time (Turner, Fielder, and Ward 2016; Shepherd et al. 2019). Gawne, Fish, and Machin (2020) advise that fostering a collaborative leadership style is the best way to support this. However, despite organisational support for learning and education, healthcare professionals may face substantial challenges when under pressure, including the demands of external regulatory authorities, and the pressures on frontline staff to deliver safe, personal, and effective care (Haywood et al. 2012; Gawne, Fish, and Machin 2020).

\section{Workplace Learning} Learning at work arises from everyday workplace activity as well as from the social relations of practice, therefore becoming a member of a professional community, and learning in the workplace are part of the same process (Lave and Wenger 1991). Successful 'communities of practice' are built according to Lave and Wenger when participation in the work is gradually increased, however they can break down if learners are not given access to the full range of activities that make up the work.

When discussing workplace learning in health care, Sholl et al (2017) described how the ward round can address professional education and patient care delivery at the same time, whereas protected learning time (PLT) and continuing professional development (CPD) keep them separate. They therefore claimed that the ward 
round can be described using Yardley et al's (2015) notion of 'experiential learning', as they 'take advantage of the existing clinical environment as the 'classroom', and therefore embrace and anticipate the difficulty of balancing health care professional education with patient care delivery' (Sholl et al. 2017:795). Discussing individual patients during ward rounds can 'increase learning opportunities, build upon opportunistic discussions and avoid undermining patient confidence when giving direct feedback to trainees' (Sholl et al. 2017:795). However, ward rounds consist of a series of complex tasks that require more than just medical knowledge, including interpersonal and communication skills.

Research has shown that learner-centred ward rounds appear to be effective in overcoming barriers to learning and can help improve educational opportunities, and thus satisfaction, for junior doctors in a busy workplace (Talbot 2000). A good ward-round structure can help maximise the learning that could take place (Acharya et al. 2015). However, despite the emphasis on clinical teaching, ward rounds often remain service driven, their educational value hindered by lack of time, noisy wards, patients not being available, reduced discussion time and an ever-increasing workload (Claridge 2011; Force, Thomas, and Buckley 2014; Talbot 2000; Bull, Mattick, and Postlethwaite 2013). It is clear then that there is a need for more research to explore the disconnection between service and education, and the resulting tensions that can develop in organisations (Cleland et al. 2018; Teodorczuk et al. 2018; Talash et al. 2020). In this paper, we report on an intervention designed to overcome the perceived service/education tensions that can bring about this separation. 


\section{The Wrap Around Project}

The balance of providing an educational environment whilst ensuring the demands and pressures of service delivery are met is a constant pressure and challenge in NHS Trusts. Gawne et al (2020) explore the requirements for front line staff to deliver safe, personal and effective care whilst meeting the demands of external regulatory authorities, emphasising the subsequent impact on the quality of training. The authors identified perceived barriers for prioritising education in the busy medical workplace and made recommendations for action, including the endorsement of collaborative leadership as key to developing an educational culture.

The present study considers the impact of what the authors refer to as the Wrap Around project that took place in a busy medical unit. A 'Wrap Around' faculty was formed in the Educational Directorate at the Trust with lead educators recruited from undergraduate, postgraduate, clinical education, non-clinical education and supporting services such as library services. Members of the faculty attended the clinical workplace to help deliver, influence and promote education in the workplace to improve the educational environment. This was done by "wrapping around" the clinical team to offer support in the form of leadership, pastoral support, coaching, mentorship and role modelling whilst raising the expectations of everyone as to how to achieve better educational experiences for all in the workplace.

The faculty followed the toolkit proposed by Gawne et al (2020) in order to identify priority areas for development within the workplace, including addressing the highlighted barriers to delivering quality medical education. The current study assesses the impact of the Wrap Around project and its contribution to cultural change in the workplace. 


\section{Method}

Semi-structured interviews were conducted to enable the researcher (RF) to probe and challenge opinions and thoughts to gain a deeper understanding of the workplace educational culture. Ethical approval was sought from Faculty of Health and Medicine Research Ethics Committee at ANON University, with additional approval from the Research and Development Unit at the Trust where the service evaluation took place.

Participants were purposively recruited from the Medical Division of a large NHS Trust in the North of England. Each participant was provided with an information sheet stating that participation was voluntary and that their interview would be recorded and transcribed with their permission. They were informed that their data would be anonymised on transcription, and their identity would be kept confidential.

The research objectives for the study were to identify the evolution of medical education within the workplace, and to explore the effectiveness of the Wrap Around intervention as perceived by staff. An initial interview guide was developed with areas to explore when investigating the structure and delivery of medical education within the specialty. The interview guide included questions such as:

- Are there clear objectives for the provision of medical education in the workplace?

- Do you have time and resources for education?

- Do you think the educational environment in the workplace is where it should be?

- What are the barriers? What is working well? 
Interviews lasted between 30 and 60 minutes and were conducted in a private area of participants' workplace.

\section{Sample Group}

Purposive sampling was undertaken by interviewing a selection of people from three groups within the Medical Division: junior doctors as learners, educational leaders who were part of the Wrap Around project, and consultant clinicians responsible for providing education (see table 1 for designations). Participants from these groups within the Medical Division were invited via a direct email to take part. Eight participants agreed to be interviewed, with many potential participants declining to participate due to time limitations. The research team decided that this purposive sample of key personnel was sufficient for the case-study design of the project.

Table 1: Participant designation

\begin{tabular}{|l|l|}
\hline Int 1 & Educator \\
\hline Int 2 & Educator \\
\hline Int 3 & Junior Doctor \\
\hline Int 4 & Junior Doctor \\
\hline Int 5 & Consultant \\
\hline Int 6 & Consultant \\
\hline Int 7 & Educator \\
\hline Int 8 & Consultant \\
\hline
\end{tabular}




\section{Analysis}

As this work was designed to be reported in case study form, and the sample was purposive involving key personnel, this influenced and determined sample size and analysis, therefore saturation was not achieved. The researcher (RF) began the analysis by coding the transcripts into major themes. The team met a number of times to discuss the analysis and visited the broad themes repeatedly in an iterative process to develop subthemes with each reading of the transcripts. This resulted in subsequent revision of the generated themes on a number of occasions until the data had been rigorously explored and consensus was met. Two 'umbrella' themes were identified in the data: enhancing balance between education and service, and challenges encountered. We will discuss each theme in the next section along with their subthemes (see table 2).

The following section will explore each analytical theme, providing quotes for illustration. To illustrate each theme, we have chosen a representative quote that we feel captures the consensus, and we have highlighted where there are conflicting quotes within that theme.

\section{Table 2: Major themes and subthemes}

\begin{tabular}{|c|c|}
\hline Major Theme & Subtheme \\
\hline $\begin{array}{l}\text { Enhancing the balance } \\
\text { between education and service }\end{array}$ & $\begin{array}{l}\text { Providing a safe space for informal learning } \\
\text { Valuing educators and learners } \\
\text { Leadership } \\
\text { Embedding the learning culture }\end{array}$ \\
\hline
\end{tabular}




\begin{tabular}{|l|l|}
\hline Challenges & Resources \\
& Motivation \\
& Personalisation \\
\hline
\end{tabular}

\section{Major theme: Enhancing balance of education with service}

Dennis et al (2014:21) define the challenges with finding the education/service balance as 'the pressures that exist or are perceived to exist between delivering service to patients and providing training'. This theme describes areas of discussion that focus on ways to overcome these challenges.

\section{Providing a safe space for informal learning}

There was agreement amongst all the interviewees that medical education should be continuous and perpetual, and should not be separate to providing patient care.

According to the interviewees, this can be achieved by making space for learning, for example:

It's part of the Duties of a Doctor by the GMC [General Medical Council] that all doctors are responsible for delivery of education. If they are a formal supervisor they'll also have time in their job to deliver certain meetings, but actually what we're talking about is making education and support and leadership part of everything we do. It should just be part of what we do. We don't do it separately it's every opportunity. (Int 5, Consultant)

This participant continued, describing the changes that have happened due to the Wrap Around intervention, as improving education coupled with service: 
[Previously,] service was such an issue that they couldn't even start thinking about the training and education until they'd sorted service out. And then even then, service was still a problem because they didn't have the leadership in place. And when we kind of addressed the leadership bit - which was through education - it kind of all came together. And I think now they get it, because by leading your team, that improves education and improves service, it's all connected. (Int 5, Consultant)

Another participant explained that daily huddles, something that was encouraged by the wrap around team, can be used for improving patient care as well as continuing learning opportunities:

So after every ward round we have a get together - a board round, essentially where we all sit down together and discuss each patient. And it's a safe environment. It's an opportunity for people to ask questions about management, etc. Obviously there's the business side of it where you need to get $x, y$ and $z$ done but there is definitely opportunities there to have a discussion about patients in a semi-informal setting. It's allocated 45 minutes. It's often an uninterrupted time as well. Everybody, all the junior doctors on the ward, attends. It's quite valuable. (Int 3, Junior Doctor)

A junior doctor agreed that the huddles provide a safe space to foster learning and communities of practice:

Often, you see consultants asking each other questions as well like, 'Why did you do that? What did you think of that?' and I think as a junior that's nice to 
see. Because it means that even they get unsure sometimes. (Int 4, Junior Doctor)

\section{Valuing educators and learners}

Junior doctors spoke about how they felt valued in the organisation, and this was beneficial to their learning. When asked what made them feel valued, one of the things they described was receiving ongoing informal feedback from consultants, for example:

When you clerk the patient and the consultant sees them, you find out whether the consultant agrees, and whether they've done anything different. So from that aspect you're learning almost instantly, especially because they do tend to see them within a few hours. . And most of the consultants do come and give you some feedback like, 'Oh, don't forget I would do this in the future' or, 'That was really good, you did a lot of good in terms of the social measure' or something. (Int 4, Junior Doctor)

Consultants were described as valuing doctors in training by recognising their level of experience and allowing them to take on more responsibility:

I know with the medical students and the PA [physician associate] students, depending on what year they're in, the consultants have an idea of what their competencies are and what they can be left to do, what teaching needs they might need, and what level they're up to. (Int 4, Junior Doctor)

This member of the educational team described some of the initiatives the Trust has put in place to support the trainees: 
How do we make the atmosphere fun for them as young people? How do we support them? And there have been a lot of initiatives. We are moving forward with a lot. I do the induction on day one and we're talking about rapport building, wellbeing, keeping yourself well. So in some ways the Trust is ahead of the curve but we really need to push that on. They're under an enormous amount of pressure and even though you say you'll have your career and all that, but we have to do the here and now. (Int 2, Educator)

A junior doctor demonstrated that the focus on teaching allows trainees to feel valued and encourages retention of staff:

I think one of the reasons I stayed on here was because we had a lot of opportunities, and everyone was really nice and I felt valued. . If you're a student here, and you have a lot of teaching, and the Trust values you as a student, it makes you want to come and work here. Because you feel like, well, they helped me as a trainee. Certainly that's how I felt, that this Trust - over a couple of other trusts that I worked at - were quite keen on teaching and developing you as a person in a new career. So that makes you want to come and work here. (Int 4, Junior Doctor)

\section{Leadership}

Leadership was described as having a major contribution to improving the learning culture, with senior managers and consultants being described as 'very supportive of teaching' and some participants commenting on the improvement in this area, for example:

Well, I think [the importance of education] is already coming down from senior management. I think it is on the agenda for senior management and that's a drive. 
I think middle management perhaps is still outcomes based/service based still and there can be a bit of disharmony there but that's what [the Wrap Around project] was for, to help empower the people delivering education so that if they do come up with any difficulties we can help support them. (Int 5, Consultant)

Educators described feeling recognized for their contributions, demonstrating the move towards a collaborative style of leadership throughout:

The [education] team are acknowledged as being knowledgeable and credible and are ensuring that education is the priority. And you can see that across the organisation. (Int 1, Educator)

\section{Embedding the learning culture}

All participants described aspects of the process of embedding a learning culture in the organization. Two participants referred to external regulators (GMC) as initiating this change in culture, that was embraced and progressed by staff:

Yeah, I think the culture has changed. And I think part of that is the drive because of the external regulation - because some people do it in order to appease the external regulation. And my feeling is that, although that might have been the initial reason for change, by activating things you can just feel a better sense of that value, of what it brings. And it actually helps with resilience as well because you feel like you get something back. It just creates a nicer culture that's happier and friendlier. And I think that's the other unintended consequence of developing an educational environment - that, yeah, everyone is just a bit happier in themselves I think. . It just becomes the standard way of doing things. (Int 5, Consultant) 


\begin{abstract}
Although the initial drive for change was perceived to come from external sources, it was felt that the learning culture had embedded itself due to the Wrap Around project as a catalyst:
\end{abstract}

That's what [the Wrap Around project] helped to do. It helped initiate that this is just the way we do things. And they'll just get on and do things now because they've been part of that [project]. And because they've got a happy bunch now, they want to keep that. They've seen that by trying different things, how it works. So it just started that off. (Int 5, Consultant)

Other consultants agreed that the experiences of medical students are improved:

I feel that there are a lot of learning opportunities within the [workplace]. And this fact is confirmed by the satisfaction of the medical students themselves. When I ask them about their feelings within the [workplace] now, they get much learning, much teaching. (Int 6, Consultant)

Junior Doctors agreed with this, describing the opportunities for learning that they have experienced, for example:

Certainly in the last 12 months, I feel like when we do have students they're letting them do more, as in they're letting them examine more patients and having a bit more time for the students if there's any interesting cases. (Int 4, Junior Doctor)

Educators mentioned that team working and support from faculty has contributed to multi-professional collaboration:

The medics that are involved in the education want to be involved in it, and we're enabling them to have the time to do it. But we're also providing support within 
clinical areas so it isn't always falling down to the medical staff to train the medical students or the trainees. We're adopting a much more of a multi-professional learning and we're wanting all the environments to be learning environments. (Int 1, Educator)

Junior doctors pointed out the need for continual improvement of this process, that ensures the involvement of everyone in education:

I think whether it's a locum consultant or a permanent one or even a registrar, I think they should be encouraged to teach no matter what grade they are. I think they should be encouraged in every situation, that you should provide some form of teaching, whether it be on the ward round or whether it be to go and examine someone. (Int 4, Junior Doctor)

\section{Major theme: Challenges to the balance}

This major theme covered challenges mentioned by the participants, including resources, personalities and the focus on formal education.

\section{Resources}

All participants mentioned time constraints as putting pressure on learning opportunities. A consideration of time pressures led to participants referring to education and service separately, as they felt the need to prioritise one over the other: I don't think there's enough time for anyone to do everything they want to do. And that includes audit, research, education. Anything other than delivering direct patient care. So I think there's a general feeling within the NHS that there's not enough time or resource really for any of us to do everything we want to. If you're 
feeling tired or everything is fraught, then the first things to go are the extra things, and education is often classed as one of them. (Int 5, Consultant)

Consultants were limited in time allowed for each patient, for example:

We have 12 [minutes per patient]. Now, if you get a very unwell patient and family we might require one hour - one patient. We may need to compromise with the other patients, with education. I think as a good physician I wish I had at least 15 or 20 minutes so that I can deliver more teaching, more talks, more discussion. We don't have time. (Int 6, Consultant)

Junior Doctors acknowledged the effect of time constraints on education:

Certainly we do a lot of practical procedures and because of time constraints it's often just left to the person who is competent to do it, rather than actually saying, you know, 'You come with me, come and see how I do it and the next one you can do.'. (Int 4, Junior Doctor)

\section{Motivation}

There was recognition that some people may not take part in teaching or not be effective at teaching:

It's in the job description, you know, when you sign up to be a doctor, the GMC say that you're going to teach other people. But I think sometimes it's personalities as well, isn't it? (Int 3, Junior Doctor)

This consultant agreed, arguing that educators' 'personalities' should not hinder the provision of training:

And, okay, some people might find it harder, but that's where training can give you the tools to still do it. You know, like the consultant who isn't very good at breaking 
bad news? Well, we can all develop it. And I think that's where we're going as a trust that it's not acceptable to say, 'Oh well, I don't do education.' Well you have to! It's part of what we do, and so it must be part of everything we do. (Int 5, Consultant)

However, a junior doctor, having acknowledged the perceptions around motivation, argued that good teaching involves being motivated to engage with junior doctors, and being willing to follow up with them:

What often happens is the junior doctor goes through the patients and then the consultant picks up the notes but doesn't necessarily get that doctor and say, 'Let's go through these patients together.' So they don't get the feedback. It's just the need to take that next step. The consultant should come along and see the same patient, get the same history, but come up and says, no, this is angina and she needs $x, y$ and $z$. So at least there's that discussion as to, 'Why did you put this? What did you think of this?' (Int 3, Junior Doctor)

Further recommendations for offering positive as well as proactive feedback were mentioned by junior doctors:

I think we're very good at providing feedback when things don't go well but we don't provide feedback necessarily when you've done well. (Int 4, Junior Doctor)

This participant went on to describe the need for feedback on individual cases:

If a patient comes in and they might move to another ward, and unless you save that patient's notes and go and have a look you'd never know what's happened. I never learn what happened to these complex patients. (int 4, Junior Doctor) 


\section{Personalisation}

Some of the junior doctors mentioned that the ward round training has reduced the informal learning tailored to their level of knowledge, for example:

I think the teaching at the bedside and on the ward round is certainly invaluable, but it's a shame really that one has had to replace the other one. So if we're discussing a patient in ward round, and it's a case of what would the next step be? The question goes out to the floor, and obviously the most junior doctor will struggle to answer that, whereas somebody who has come with their core medical training will find that a lot easier to answer. So rather than it being different questions asked for different abilities it's just a generic thing. But, when a consultant goes with one junior doctor to the bedside, then you get that one-toone teaching that's directed at your level. I think it's quite powerful and it's very individualised as well. It addresses your own learning needs. (Int 3, Junior Doctor)

This issue was flagged up by another Junior Doctor who referred to the need to focus on the strengths of the whole group:

I think we're definitely valued. But, I think there could be a lot more focus on the group development as a whole and recognising that there are different grades of doctors here. We have a lot of physicians, nurse practitioners, etc. and everybody's needs are different. Sometimes the teaching can feel a bit generic and it's not necessarily personalised, which is disappointing I think for some of the more senior doctors. (Int 4, Junior Doctor) 


\section{Discussion}

Sholl et al's synthesis of research concerning balancing healthcare education with care of patients (2017) found that the most positive common outcome of improving the learning culture in the workplace was that both patient care and education improved simultaneously. In agreement with their observations, our participants reflected that embedding a learning culture has worked to improve both patient care and education on the wards. Hussain, Rossi, and Rynne (2019) advise that successful learning cultures are holistic and that internal competitiveness should be minimised amongst learners. They recommend greater integration of inter-professional interactions to maximise learning, analogous to our participants' notions of personalisation and collaborative leadership. These recommendations correspond to Lave and Wenger's (1991) notions of 'communities of practice', where learners work closely with 'near peers' and are able to actively participate in all aspects of the work. Sholl et al's (2017) review found that the main challenges to the development of a successful learning culture were time constraints and trainees feeling undervalued, and these issues were flagged as challenges by our participants.

We agree with Sholl et al, that education needs to be fully integrated into the workplace at organisational, departmental, and individual levels in order for it to be translated into high quality service delivery and patient care - and that the availability of time, cover, and appropriate funding should be put in place in order for this to happen. Integration of education can be achieved through fostering effective collaborative leadership and empowerment throughout (Rushmer et al. 2004; Meeuwissen et al. 2021; Stewart et al. 2019; Shepherd et al. 2019), as well as 
advancing organisational support for active participation in learning (Acharya et al. 2015).

Sholl et al (2017) found that trainers were often not confident that an adequate balance of service delivery and education could realistically be maintained, coupled with a perception by junior doctors that service provision is generally prioritised over their training. Sholl et al argue that without adequate recognition for workplacebased education, learners may experience suboptimal career progression, plus higher attrition rates. We found little evidence of these attitudes - participants in our case study recognized the relationship between effective service and education, whilst also acknowledging the associated potential for reduced staff turnover when the balance is addressed.

When analyzing our themes conceptually, we found that embedding a culture of learning together in the workplace can be achieved by working on balancing the conflicts between service and education. We argue that this may be done by using interventions that address the toolkit proposed by Gawne et al (2020), targeting leadership, attitudes and systems at all levels throughout the organization. Whilst the Wrap Around project managed to improve many areas of educational culture, bringing about much change, we noticed that service and education are still referred to as separate (albeit interrelated) issues, something that has been highlighted by Cleland et al (2018) and Sanfey et al (2011). It is important for services to recognize that these activities are mutually dependent in order to strengthen perceptions of the importance of patient care as an essential component of competency (Sanfey et al. 2011). 


\section{Conclusion}

Whilst initial motivation for changes in educational culture may originate outside healthcare organisations, we have identified that when support for education is provided at all levels, this can have the effect of improving staff morale, retention, and in turn, better patient care. Although this case study is situated in one NHS Trust, the results have been remarkable and are continuing to progress. We recommend further research that explores creative initiatives, that build on the embedded educational culture and work to reduce the perceived distinction between education and service (Morris and Swanwick 2018).

\section{Implications for practice}

- Fostering an educational culture relies on finding a way to balance the tensions between service and education.

- Ways to enhance this balance can be through interventions that involve all levels of the organization.

- Embedding a culture for workplace-based learning involves valuing learners and educators, supporting collaborative leadership, and recognizing the importance of time and safe spaces for learning.

\section{References}

Acharya, Vikas, Amir Reyahi, Samuel M Amis, and Sami Mansour. 2015. "Do "traineecentered ward rounds" help overcome barriers to learning and improve the learning satisfaction of junior doctors in the workplace?" Advances in medical education and practice 6: 583.

Bull, Stephanie, Karen Mattick, and Keith Postlethwaite. 2013. "Junior doctor decision making: isn't that an oxymoron?'A qualitative analysis of junior doctors' wardbased decision-making." Journal of Vocational Education \& Training 65 (3): 402421.

Claridge, Andrew. 2011. "What is the educational value of ward rounds? A learner and teacher perspective." Clinical Medicine 11 (6): 558. 
Cleland, Jennifer, Ruby Roberts, Simon Kitto, Pia Strand, and Peter Johnston. 2018. "Using paradox theory to understand responses to tensions between service and training in general surgery." Medical education 52 (3): 288-301.

Dennis, Ashley A, Jennifer A Cleland, Peter Johnston, Jean S Ker, Murray Lough, and Charlotte E Rees. 2014. "Exploring stakeholders' views of medical education research priorities: a national survey." Medical education 48 (11): 1078-1091.

Eraut, Michael. 2011. "Informal learning in the workplace: evidence on the real value of work-based learning (WBL)." Development and Learning in Organizations: An International Journal 25 (5): 8-12.

Force, Jade, Ian Thomas, and Frances Buckley. 2014. "Reviving post-take surgical ward round teaching." The clinical teacher 11 (2): 109-115.

Gawne, Suzanne, Rebecca Fish, and Laura Machin. 2020. "Developing a workplace-based learning culture in the NHS: aspirations and challenges." Journal of Medical Education and Curricular Development 7: 2382120520947063.

Haywood, Hannah, Helen Pain, Sarah Ryan, and Jo Adams. 2012. "Engagement with Continuing Professional Development Development of a Service Model." Journal of allied health 41 (2): 83-89.

Hussain, Aman, Tony Rossi, and Steven Rynne. 2019. "Learning in the ED: chaos, partners and paradoxes." Journal of Workplace Learning 31 (6): 361-376.

Lave, Jean, and Etienne Wenger. 1991. Situated learning: Legitimate peripheral participation. Cambridge university press.

Meeuwissen, Stephanie N. E., Wim H. Gijselaers, Tiemen D. van Oorschot, Ineke H. A. P. Wolfhagen, and Mirjam G. A. oude Egbrink. 2021. "Enhancing Team Learning through Leader Inclusiveness: A One-Year Ethnographic Case Study of an Interdisciplinary Teacher Team." Teaching and Learning in Medicine: 1-11. https://doi.org/10.1080/10401334.2021.1887738.

Morris, Clare, and Tim Swanwick. 2018. "From the workshop to the workplace: Relocating faculty development in postgraduate medical education." Medical teacher 40 (6): 622-626.

Mulder, Hanneke, Edith Ter Braak, H Carrie Chen, and Olle Ten Cate. 2019. "Addressing the hidden curriculum in the clinical workplace: A practical tool for trainees and faculty." Medical Teacher 41 (1): 36-43.

Rushmer, Rosemary, Diane Kelly, Murray Lough, Joyce E Wilkinson, and Huw TO Davies. 2004. "Introducing the Learning Practice-III. Leadership, empowerment, protected time and reflective practice as core contextual conditions." Journal of evaluation in clinical practice 10 (3): 399-405.

Sanfey, Hilary, Joe Cofer, Jonathan R Hiatt, Matthew Hyser, Colleen Jakey, Stephen Markwell, John Mellinger, Richard Sidwell, Douglas Smink, and Stephen Wise. 2011. "Service or education: in the eye of the beholder." Archives of Surgery 146 (12): 1389-1395.

Shepherd, Lisa, Kori A LaDonna, Sayra M Cristancho, and Saad Chahine. 2019. "How medical error shapes physicians' perceptions of learning: an exploratory study." Academic Medicine 94 (8): 1157-1163.

Sholl, Sarah, Rola Ajjawi, Helen Allbutt, Jane Butler, Divya Jindal-Snape, Jill Morrison, and Charlotte Rees. 2017. "Balancing health care education and patient care in the UK workplace: a realist synthesis." Medical education 51 (8): 787-801.

Stewart, Victoria, Matthew Campbell, Sara S McMillan, and Amanda J Wheeler. 2019. "Postgraduate work-based learning: a qualitative study." Higher Education, Skills and Work-Based Learning 9 (4): 637-649.

Talash, Khojasta, Lorraine Corfield, Natalie Latcham, Claire Lavelle, Richard a Williams, and Laura L. Machin. 2020. "Exploring UK foundation doctors' perceptions surrounding raising concerns in the workplace." Journal of Vocational Education \& Training: 1-15. https://doi.org/10.1080/13636820.2020.1792535. 
Talbot, Martin. 2000. "An interview study of the working ward round as an instrument of experiential learning in postgraduate medical education: a preparatory exploration." Journal of Vocational Education and Training 52 (1): 149-159.

Teodorczuk, Andrew, Rola Ajjawi, Stephen Billett, Joanne Hilder, and Christy Noble. 2018. "The service/teaching tension: a window into the soul of a hospital."

Turner, Teri L, Elaine Fielder, and Mark A Ward. 2016. "Balancing service and education in residency training: a logical fallacy." JAMA pediatrics 170 (2): 101-102.

Yardley, Sarah, Elizabeth Cottrell, Eliot Rees, and Joanne Protheroe. 2015. "Modelling successful primary care for multimorbidity: a realist synthesis of successes and failures in concurrent learning and healthcare delivery." BMC family practice 16 (1): 1-21. 